\title{
NUMERALS, POSITIONALITY, AND REFERENCE FIXING. REPLY TO VIVANCO ${ }^{1}$
}

\author{
MARIO GÓMEZ-TORRENTE \\ https:/ / orcid.org/0000-0002-5957-6544 \\ Instituto de Investigaciones Filosóficas \\ Universidad Nacional Autónoma de México (UNAM) \\ Mexico City \\ Mexico \\ mariogt@unam.mx
}

\section{Article info}

CDD: 401

Received: 01.10.2020; Accepted: 17.10.2020

https://doi.org/10.1590/0100-6045.2020.V43N4.MZ

\section{Keywords \\ Numerals \\ Positionality \\ Reference}

\begin{abstract}
Melisa Vivanco objects to my theory of the Arabic numerals in Roads to Reference that the reference fixing procedure that I postulate doesn't exploit the morphological structure of the Arabic numerals, but it should. Against Vivanco, I argue that the procedure in question does exploit the morphological structure of the numerals in an essential way.
\end{abstract}

A common metasemantic view of the decimal Arabic numerals that we use all the time is that their referents (or at least those of the complex ones) are fixed in a typical speaker's idiolect by the (either synonymous or "merely"

\footnotetext{
${ }^{1}$ Support from the research project no. PIDPID-107667GB-I00 of the Spanish Ministry of Science and Innovation is gratefully acknowledged.
} 
reference-fixing) polynomials that detail the contribution of each digit in a complex numeral as the multiple of the corresponding power of ten. For example, “765" gets its reference fixed by the polynomial description " $5 \cdot 10^{0}+$ $6 \cdot 10^{1}+7 \cdot 10^{2}$ ". I criticize these and other related proposals in Roads to Reference before presenting my alternative view, on which the reference of complex Arabic numerals is "merely" fixed (in a typical speaker's idiolect) by comparatively unsophisticated descriptions that single out the referents of later numerals by means of operations (e.g., simple sums) on the referents of earlier numerals in the natural series of Arabic numerals; for example, in the simplest case, in general the reference of a sufficiently "big" decimal Arabic numeral is fixed as the number that results from adding one to the referent of its preceding decimal Arabic numeral. Here the series of numerals is postulated to be generated "lexicographically" in the mind of the speaker, independently of semantic interpretation. On the other hand, the reference of some of the "small" numerals, such as some of the digits, is fixed by a species of descriptive ostension ("this number") of little bunches or mental models of the corresponding small cardinalities. All these descriptions use the concept of "number"; in the book (and also in Gómez-Torrente (2015)) I also argue that several ideas inherent in the common conception of number single out the finite plural cardinality properties as the things to which "number" applies, and hence that presumably the natural numbers are nothing but the plural cardinality properties. Another important feature of the proposal is that, if reference fixing for the numerals typically works this way, speakers can be said to be in a straightforward epistemic contact with the numbers: since these are the plural cardinality properties, and given that it's natural to think that one kind of close epistemic contact with a property is provided by the ability to tell in a non- 
sophisticated way when the property applies from when it does not, speakers can be said to be in close epistemic contact with the numbers because (1) they know how to count, i.e. they know how to generate a suitable series of interpreted numerals and put it in correspondence with the bunch of things to be counted, thereby ascribing the relevant cardinality property of the bunch, and (2) they can easily tell, once they have counted two bunches and assigned two corresponding numerals to them, whether the two bunches have the same or a different cardinality property (at least for bunches which are not too big).

Melisa Vivanco (2020) likes several features of this picture, which she actually seeks to reinforce at a number of points, using her own considerations; in particular, she embraces the identification of the numbers with the plural cardinality properties. Her criticisms focus on the idea that the picture doesn't do enough justice to the intuitions behind the polynomial account. In Roads to Reference I criticize the polynomial account with several arguments. One is that children seem to assign an interpretation to the numerals (which they have previously learned to generate in a more or less "mindless" way) well before they can make sense of polynomials or of exponentiation, or even full sense of multiplication. Another argument is that, to the extent that we could entertain the epistemic possibility that exponentiation or even multiplication might somehow be problematic for sophisticated mathematical reasons (like those given by some radical constructivists), the way in which the Arabic numerals get their referents appears to be susceptible of being seen as "detached" from the standard polynomials, which feature representations for such operations (see Gómez-Torrente (2019), 120ff.): intuitively, "765" would still be meaningful (in a typical speaker's idiolect) if exponentiation or even multiplication turned out not to be bona fide operations for some sophisticated 
mathematical reason: intuitively it seems to be enough that "765" can play the role of numbering pluralities of 765 elements (and that its fellow numerals do their jobs as well).

My proposal is an attempt to get a picture of the situation that respects these facts and intuitions (and others). On the proposal, the principle that generates the series of Arabic numerals, i.e. basically the lexicographic procedure, is already essentially mastered by typical users of the numerals before these acquire an interpretation or reference in their idiolects. For each generated numeral, this interpretation is provided "from below": in the simplest case, a non-initial numeral gets its referent as the result of adding one to the referent of the preceding numeral (which has "already" been generated and provided with a reference)-adding one being simply the operation that yields the property of ascribing one more element than a given cardinality property. (Surely the operation of adding one cannot be imagined to be possibly (epistemically) problematic: if it were, the whole idea of number would be problematic as well.) Reacting to this, Vivanco says:

The procedure may seem adequate for consecutive numerals whose morphology does not change significantly after the transition by adding 1 of the corresponding numbers. Consider for example, '764' and ' 765 ' (since ' 5 ' is the numeral that follows ' 4 ' in the natural order, whose referent is obtained from the fact that 5 is the number greater by one than 4), we have a straightforward account for the reference of ' 765 ' in terms of their constituents. Nonetheless, the account seems to lack an explanatory element for cases like '999' and '1000' whose morphologies (determined by 
the constituents) differ more substantially. In my opinion, Gómez-Torrente's account underestimates the fact that Arabic numeral systems are positional, which is a salient aspect regarding their morphology. I think that unsurprisingly, this property is not only associated with a relevant characteristic of numbers, but it also plays an important role in how Arabic numerals manage to refer to numbers.

Thus, according to Vivanco, the reference fixing procedure in my picture "seems to lack an explanatory element for cases like '999' and '1000"', and this difficulty appears to immediately lead her to postulate that the fact that Arabic numerals conform a positional system must somehow be crucial to the fixing of their referents. The "relevant characteristic" to reference fixing that positionality brings out is that numbers can be partitioned into summands that correspond to the summands in the standard polynomial representation. In the case of " 765 ", these summands are " $5 \cdot 100 "$ ", " $6 \cdot 10^{1 "}$ " and " $7 \cdot 102 "$ ", but if we accept the "detachment" arguments mentioned above concerning exponentiation and multiplication, we might propose (and this is essentially Vivanco's proposal) that the relevant summands are " 5 ", " 60 " and " 700 ". The natural way to reflect this relevance is to go back to a (slight modification of the) polynomial proposal on which the reference of, say, " 765 " is given by the summation " $5+60$ +700 ". Vivanco summarizes the virtues of the picture thus:

The cardinality property (number) of a plurality can be split up according to the following fact: the plurality can be partitioned 
into smaller pluralities, each of which corresponds to an Arabic numeral. The relation between the cardinality properties of the sub-pluralities and the cardinality property of the total plurality is associated with the relation between each of the constituents and the complex Arabic numeral that corresponds to the total plurality. The way these partitions can be selected is reflected in the morphology of the corresponding Arabic numeral. In particular, in the value that each constituent has, according to its position.

As she notes, Vivanco is seeking to give its due place to the desideratum I mention in Roads to Reference, that "the Arabic numerals get interpreted via a general procedure which exploits their morphological constitution" (GómezTorrente (2019), 122). I obviously agree with this, but I disagree with Vivanco's claim that the reference-fixing procedure I postulate does not satisfy the desideratum. As we have seen, her reason for thinking this is that, even if "764" and "765" have similar morphologies, and in particular differ only in that the last digit of the latter refers to a number that comes from the number referred to by the last digit of the former by the operation of adding one to it, "999" and " $1000 "$ have a very different morphology, and it's just not clear how the referent of the latter is connected via morphology to the referent of the former. But this is not a valid reason. The key to seeing why it's not is to see what the lexicographic procedure of numeral generation involves. The procedure is simple: first go the digits " 0 ," "1", “2", “3”, “4”, “5”, “6”, “7”, "8” and " 9 " in this very order; and then, for sequences of two or more digits not beginning with zero, a sequence of digits $\mathrm{d}_{1} \mathrm{~d}_{2} \ldots \mathrm{d}_{\mathrm{n}}$ comes before a sequence $\mathrm{d}_{1}{ }^{\prime} \mathrm{d}_{2}{ }^{\prime} \ldots \mathrm{d}_{\mathrm{k}}$ ' iff $\mathrm{n}$ is less than $\mathrm{k}$ or $\mathrm{n}=\mathrm{k}$ and, 
at the first $i$ where $d_{i}$ and $d_{i}^{\prime}$ differ, $d_{i}$ comes before $d_{i}$ ' in the list of the digits. This directly implies that "999" is the last numeral of three digits, and, since the first numeral of four digits is "1000", that "1000" immediately follows "999" in the series of decimal Arabic numerals. That is, given the definition of the mentioned (essentially) lexicographic ordering, that "1000" immediately follows "999" in the series is something that immediately follows from their morphologies. Then, provided "999" has a referent, "1000" will ipso facto get one as the number that results from adding one to the referent of "999". (But "1000" may get its referent via a different summation once the referents of earlier numerals have been fixed; see below.) Surely the procedure exemplified in this case interprets the numerals via a procedure which exploits their morphological constitution.

If we are to avoid recourse to exponentiation or multiplication, there are serious difficulties for a modification of the polynomial proposal in the spirit of Vivanco's. In order for the reference of " 765 " to be given by the summation " $5+60+700$ " in a typical speaker's idiolect, "5", "60", and "700" must already have a reference in this idiolect. Assuming " 5 " is allowed to get a reference by some independent mechanism, such as the mechanism of descriptive ostension mentioned above, how do " 60 " or "700" get a reference, given that we are supposing that they cannot get it via descriptions such as " $6 \cdot 10$ " and " $7 \cdot 102$ " or " $6 \cdot 10$ " and " $7 \cdot 100$ "? Note that even bracketing the appearance of multiplication, the use of " $6 \cdot 10$ " and " $7 \cdot 100$ " presupposes that " 10 " and " 100 " have been provided with a referent; but how is this supposed to be provided? The proposal in Roads to Reference evades these problems with its postulation that the numerals are provided with a reference in terms of sums. In a typical speaker's idiolect (a typical child), "10" may get its referent 
as " $9+1$ ", and " 60 " may get its referent as the child learns to generate the series of numerals up to " 60 " by repeated applications of the operation of adding one, or perhaps by other repeated sums, such as " $10+10+10+10+10+$ 10". Similarly for " $100 "$ ". "700" may then again get its referent by repeated applications of the operation of adding one or by other repeated sums, such as "100 + 100 + 100 $+100+100+100+100 "$. (And similarly for "1000") (Note that all this is not to say that absolutely no speaker uses, say, multiplication or exponentiation in descriptive associations with e.g., "700"; it's just to say that if this happens, the speaker already has a more basic "backup" interpretation of " 700 " which provides it with a reference in terms of sums.)

Another worry Vivanco has is that the reference-fixing procedure proposed in Roads to Reference as the one at work with typical speakers is one that would also work for systems of numerals other than that of the decimal Arabic numerals:

what does [the procedure of adding as reference-fixing] have to do with the morphological components and meanings of Arabic numerals? There seems to be no reason to think that the procedure would not work for other numbering systems that are essentially different from Arabic numerals. In particular, for systems in which numerals have a substantially different morphology. If so, it is very unclear that [numerals get their interpretations by means of a general procedure which exploits in some way their morphological constituents]. 
That, on the contrary, the reference-fixing procedure I propose exploits the morphology of the decimal Arabic numerals was made clear above. (And the same could be said of the procedure as applied to the Arabic numerals in other bases, and of the procedure as applied to other complete numbering systems.) But is it somehow damaging to the proposal that "the procedure would work for other numbering systems"? I cannot see why. Vivanco's reason seems to have to do with the idea that the characteristics of the procedure ought to have by themselves the implication that the procedure's having been used is what makes possible the existence of de re attitudes involving the decimal Arabic numerals. (In the book I note and make use of the fact that typical speakers entertain de re attitudes toward relatively big numbers via their decimal Arabic numerals, but not via their corresponding polynomials.) She says, for example, that "the fixing-the-reference procedure must lead to an ontology and epistemology that accounts for our de re attitudes toward numbers through the use of Arabic numerals" and asks us to

consider a non-positional system (one in which the position a symbol occupies in the numeral bears no relation to its value). For instance, the system where ' 1 ' stands for the number one, '11' stands for the number two, '111' stands for the number three, and so on. The procedure offered by Gómez-Torrente succeeds in accounting for how the numerals in this system get their references fixed. The speaker picks out the referent of '1111111111' in virtue of the fact that the numeral ' 1 ' refers to the number one and the ability to recognize that '1111111111' follows to '111111111' because its referent (the number ten) is the 
number greater by one than the referent of '111111111', which has been already fixed by the same procedure. That which constitutes [the procedure] is apparently all that is needed to explain how almost any system whose numerals refer to numbers manages to do so. Certainly, such numerals do not seem to entertain de re attitudes toward the number 102 as the numeral '102' does in the positional decimal Arabic system. (Or as the numeral '1212' allegedly would do if we had been trained in the Arabic numeral system in base 4).

The problem with this line of reasoning lies in Vivanco's assumption, which obviously she attributes also to me, that the description of the reference-fixing procedure ought to imply by itself that, when the procedure has been applied, de re attitudes involving the numerals (for which referents have been fixed through the procedure) automatically become possible. But I never make such assumption, and I am puzzled as to why Vivanco makes it. My position in the book is that the reference-fixing procedure does fix the plural cardinality properties as referents of the numerals (and indeed, that the procedure could also be used in order to fix the referents of the numerals in a non-positional but complete system like the one described by Vivanco, in a positional base 2 Arabic notation, or in a positional base 100 Arabic notation). De re epistemic contact with those properties via the numerals is then possible by the acquisition of the abilities to tell in principle when the properties apply to a bunch of things via counting with the numerals and to tell when a bunch has more or fewer things than another via comparison of the corresponding numerals that have been assigned to them by counting (at 
least for bunches which are not too big). But the acquisition of these abilities is independent from the reference-fixing procedure, in the sense that the application of the latter does not necessitate the former. Undoubtedly many additional factors have to be in place in order for those abilities to be acquired with respect to the relevant numerals, including an appropriate cognitive wherewithal. Human cognitive capacities are not suited to an acquisition of those abilities for a system of numerals as strings of “1"'s, nor for a base 2 system (we just don't seem to be able to effortlessly compare two moderately long strings of " 1 "'s, or two moderately long strings of " 1 "”s and " 0 "”s), nor for a base 100 system (we just don't have the memory required to remember effortlessly the interpretation of 100 basic digits). Our standard base 10 notation appears to achieve a very good compromise from the point of view of our cognitive capacities, both with respect to its number of basic digits and with respect to the distinguishability and comparability of moderately long strings of those digits. People acquire without excessive effort the mentioned abilities for the decimal Arabic numerals, and that's presumably one of the reasons why this notation has eventually become standard. But the acquisition of these abilities is a further step that goes beyond the mechanism of reference fixing.

Note that Vivanco's reference-fixing proposal does not live up to her own standards. If all I have is an instruction to assign an appropriate "partitional" summation to, say, each numeral in the system of base 2 strings of " 0 "'s and " 1 "'s, or to each numeral in a system of base 100 numerals, then, even assuming for the sake of argument that this fixed the referents of these numerals as the corresponding plural cardinality properties, I would not necessarily have acquired the abilities to count easily with these numerals and to compare effortlessly any two of them; in fact, typical 
human cognitive capacities appear to be simply unfit for these tasks. ${ }^{2}$ Mastering a reference fixing procedure cannot do for me everything I want of the numerals, regardless of whether the procedure is a version of the polynomial proposal or something along the lines of the proposal in Roads to Reference.

\section{REFERENCES}

Gómez-Torrente, M. (2015), "On the Essence and Identity of Numbers", Theoria 30, 317-29.

Gómez-Torrente, M. (2019), Roads to Reference. An Essay on Reference Fixing in Natural Language, Oxford University Press, Oxford.

Vivanco, M. (2020), "Referential Uses of Arabic Numerals", Manuscrito, this issue.

2 Vivanco would want to claim, in any case, that only the numerals in a positional system can be involved in de re attitudes toward the numbers via numerals. But I don't see any reason why some beings with cognitive capacities different from those of typical humans could not entertain de re attitude attitudes toward numbers via long strings of " 1 "”s, say. As far as I can tell, this might require only greater memory and computation capacities than typical human brains have. 